\title{
programa de filosofía para niños como propuesta de educación moral: análisis comparado con otros enfoques de la educación moral
}

\author{
adolfo agundez-rodriguez ${ }^{1}$ \\ université du québec à montréal, canada
}

resumen

Lo que trataremos de demostrar en el presente artículo es que la propuesta de Filosofía para niños $(\mathrm{FpN})$, pensada holísticamente $\mathrm{y}$, en particular, en su dimensión ética, puede ser concebida como un programa de educación moral. Para ello, después de hacer un breve repaso de los fundamentos epistemológicos que se encuentran en la base del programa Filosofía para niños con respecto a la educación moral, pasaremos a comparar la vertiente ética de dicho programa con otros enfoques de la educación moral (el enfoque de la Clarificación de valores, el de la solicitud, el narrativo y el de las éticas discursiva, cordial y de la reconstrucción). A fin de completar nuestro análisis, en la parte final de nuestro trabajo, presentamos una crítica a la propuesta de Lipman, lo cual, más que restarle validez, puede servir para proyectar este enfoque de educación moral más allá de sus propios límites. Sostenemos que, tomada en su apuesta de educación moral, la Filosofía para niños es una propuesta integradora y original. Integradora, en el sentido de que es una propuesta que reúne los elementos esenciales presentes en los principales enfoques contemporáneos de la educación. Original, por promover el desarrollo de un pensamiento de orden superior, crítico, creativo y cuidante, como necesario a la formación permanente del sujeto moral.

palabras clave: filosofía para niños; educación moral; educación comparada.

\section{programa de filosofia para crianças como proposta de educação moral: análises comparadas com outros enfoques de educação moral.}

resumo

O que trataremos de demonstrar no presente artigo é que a proposta de Filosofia para crianças $(\mathrm{FpN})$, pensada holisticamente e, em particular, em sua dimensão ética, pode ser pensada como um programa de educação moral. Para isso, depois de fazer uma breve revisão dos fundamentos epistemológicos que se encontram na base do programa Filosofia para crianças com relação à educação moral, passaremos a comparar a vertente ética desse programa com outros enfoques da educação moral (o enfoque da Clarificação de valores, o do pedido, o narrativo e das éticas discursivas, cordial e da reconstrução). A fim de complementar nossas análises, na última parte do nosso trabalho, apresentamos uma crítica à proposta de Lipman, a qual, ao invés de diminuir sua validade, pode servir para projetar esse enfoque de educação moral além de seus próprios limites. Sustentamos que, tomada em sua proposta de educação moral, a Filosofia para crianças é uma proposta integradora e original. Integradora no sentido de que é uma proposta que reúne os elementos essenciais presentes nos principais enfoques contemporâneos da educação.

1 adolfo.agundez@trudeaufoundation.net

childhood \& philosophy, rio de janeiro, v.14, n. 31, set.-dez. 2018, pp. 671-695 issn 1984-5987 
programa de filosofía para niños como propuesta de educación moral: análisis comparado con otros enfoques de la educación moral

Original por promover o desenvolvimento de um pensamento de ordem superior, crítico, criativo e zelador como necessário à formação permanente do sujeito moral.

palavras-chave: filosofia para crianças; educação moral; educação comparada.

\section{philosophy for children program as a proposal of moral education: analysis compared with other approaches to moral education}

\section{abstract}

What we will try to demonstrate in the present article is that the proposal of Philosophy for Children (P4C), regarded holistically and, in particular, in its ethical dimension, can be thought of as a program of moral education. In order to do this, after a brief review of the epistemological foundations that are at the base of the Philosophy for Children program with respect to moral education, we will compare the ethical aspect of this program to other approaches to moral education (the approach of the clarification of values, that of request, narrative and that of discursive, cordial and reconstruction ethics). Finally, to complete our analysis, we will present a critique of Lipman's proposal, which, far from diminishing its validity, can be used to project this approach to moral education beyond its own limits. We maintain that, taken in its commitment to moral education, Philosophy for Children is an integrative and original proposal. Integrative, in the sense that it is a proposal that brings together the vital elements present in the main contemporary approaches to education. Original, for promoting the development of a higher order, critical, creative and caring thought as necessary to the permanent formation of the moral subject.

keywords: philosophy for children; moral education; comparative education. 
programa de filosofía para niños como propuesta de educación moral: análisis comparado con otros enfoques de la educación moral

\section{introducción}

El programa de Filosofía para niños $(\mathrm{FpN})$ es una propuesta educativa de la persona en toda su integridad y, por ende, lo es también de la persona como sujeto moral. Si bien, de acuerdo con Rubio (2000), la educación moral parece a primera vista una "tarea imposible atrapada entre la socialización adaptativa y el individualismo subjetivista" (p. 65), creemos que la propuesta holística de FpN en su vertiente ética ${ }^{2}$ permite superar los límites señalados por el autor y se ajusta a su definición de educación moral:

es posible plantearse la educación moral como una tarea de construcción dialógica de ciertas normas y pautas de conducta mediante las que la persona puede elaborar soluciones creativas y responsables a los dilemas [cuestiones y problemas] axiológicos que se le presentan de continuo, tanto en su ámbito privado como en el público (social y político) [...] la persona ha de ser capaz de elegir autónomamente entre las alternativas o dilemas morales que se le ofrecen, pero su solución ha de ser "enseñable", esto es, ha de poder justificarse mediante argumentación intersubjetiva racional (p. 66).

Esta concepción, compatible con el pluralismo moral e incompatible con el relativismo axiológico, es la defendida desde los principales enfoques contemporáneos de la educación moral. Lo que trataremos de demostrar en el presente artículo es que la propuesta de $\mathrm{FpN}$ se ajusta a dicha definición, lo cual le confiere validez como programa de educación moral. Para ello, tras hacer un breve repaso de los fundamentos epistemológicos en la base de la propuesta de FpN para la educación moral, pasaremos a comparar la vertiente ética del programa con otros enfoques de la educación moral. A fin de completar nuestro análisis, en la parte final de nuestro trabajo, presentamos una crítica a la propuesta de Lipman, lo cual, más que restarle validez, puede servir para proyectar este enfoque de educación moral más allá de sus propios límites.

\section{fundamentos epistemológicos de la propuesta de fpn para la educación moral}

Según Daniel (1992), la propuesta de FpN de Lipman queda inscrita, de manera general, en lo que ella denomina como neo-pragmatismo filosófico, aunque con diferencias notables respecto a la línea neo-pragmática de Rorty (1996) o Putnam (1999). Además, al referirse concretamente a la educación moral, Daniel (Ibid.) afirma que este carácter pragmático del pensamiento de Lipman coincide cien por cien con el pensamiento moral en Dewey (1909/1969). Según Miranda

${ }^{2}$ En el presente texto emplearemos el término ética en el sentido de Filosofía moral, de ciencia que se ocupa de lo moral. 
programa de filosofía para niños como propuesta de educación moral: análisis comparado con otros enfoques de la educación moral

(1991), una de las ideas centrales del pensamiento moral de Dewey es que el conocimiento moral está integrado en el conocimiento científico, lo cual nos permite afirmar con el autor norteamericano el carácter científico del conocimiento moral:

La moral, considerada como ciencia, es un saber empírico y objetivo, pero hipotético y en evolución, porque la vida es un proceso en movimiento, en donde las viejas verdades morales se quedan inservibles. Como ocurre en las demás ciencias, no cabe hablar tampoco de principios y leyes morales inmutables, pues estos tienen un marcado carácter hipotético y son confirmados o falsados en la acción y experimentación (MIRANDA, Ibid., p. 9).

Esta idea, que se prolonga en el pensamiento lipmaniano, se traduce en una propuesta de educación moral que toma forma de investigación moral, según veremos más adelante. Según Daniel (1992), esta concepción de la educación moral, en Lipman como en Dewey, se asienta en los siguientes puntos comunes: en primer lugar, ambos filósofos defienden la exigencia de una educación moral holística, basada en el desarrollo de las capacidades tanto cognitivas, como intelectuales y afectivas; en segundo lugar, para ambos la educación moral se realiza a través de la experiencia bajo los principios de continuidad e interacción; por último, tanto Lipman como Dewey entienden como valores principales la utilidad, la responsabilidad y la solidaridad, siendo la empatía (o simpatía, más bien, en Dewey) la base del comportamiento moral; una empatía-simpatía que supone dos actitudes fundamentales, la apertura y la solidaridad.

Sin embargo, y en contra de lo que cabría suponer, el pensamiento de Lipman no es una copia del de Dewey en materia moral. Lipman retoma la teoría moral de Dewey para actualizarla, fundamentalmente en lo que se refiere a la puesta en práctica de la educación moral. En efecto, la esencia misma del programa de FpN es moral (Daniel, Ibid.). Y, si bien Dewey ha sido fecundo a la hora de pensar el desarrollo moral, no lo ha sido tanto en el momento de orientar, como en el caso de Lipman, la educación moral.

\section{programa de fpn en su vertiente moral: análisis comparativo con otros enfoques contemporáneos de la educación moral}

En este apartado presentamos un análisis comparado de la propuesta del programa de FpN para la educación moral. En primer lugar, examinaremos el programa a la luz de los principales enfoques contemporáneos de la educación moral: el de la Clarificación de valores, el de la solicitud, el enfoque narrativo y el de las éticas discursiva, cordial y de la reconstrucción. En segundo lugar, analizaremos un elemento original y clave en la propuesta ética lipmaniana: el 
pensamiento cuidante, como pensamiento comprometido con la búsqueda del valor.

\section{fpn y clarificación de valores: el carácter holístico de la educación moral}

Según Lipman y al. (1998),

es bastante común plantear el problema del desarrollo moral del niño del siguiente modo: la educación moral debe ser interpretada como una manera de lograr que los niños se adapten a los valores y costumbres de la sociedad a la que pertenecen; o bien, como una manera de liberar a los niños de esos valores y costumbres, a fin de que lleguen a ser individuos autónomos y libres (...). Considerar a los individuos o a la sociedad como innatamente buenos o malos es cerrar de antemano la posibilidad de decidir a través de la investigación quién es responsable de cada situación y cómo puede ser mejorada. En este sentido, toda afirmación dogmática acerca de la sociedad o la naturaleza del individuo cancela la investigación (LIMPAN y al., 1998, p. 266).

Lejos de las concepciones reductoras de la educación moral, Lipman plantea una educación moral como contenido y como proceso (Daniel, 1992; García Moriyón, 1998). La educación moral como contenido haría referencia al sentido normativo de la moral y echaría mano de una serie de convenciones, reglamentos y normas, así como de criterios para evaluarlos. Sin embargo, esta educación moral como contenido se queda en su ámbito más superficial sin el concurso de la educación moral como proceso, es decir, como educación a la "buena vida humana", en palabras de Savater (1992), entendida como búsqueda siempre inconclusa de la individualidad y del sentido de la propia vida, así como centrada en la práctica, en el uso de la libertad y en la reciprocidad: "darse la buena vida no puede ser algo muy distinto a fin de cuentas de dar la buena vida [a otros]" (Ibid., p. 79). Además, al concebir la educación moral como proceso, Lipman se encuentra de nuevo con Dewey (1922/1964), para quien el único fin de la educación moral es dicho progreso y el crecimiento moral consecuente. Miranda (1991) rescata para nosotros esta cita de la obra de Dewey (Ibid.), que no deja dudas al respecto:

el fin deja de ser un término o límite que hay que alcanzar; se convierte en el proceso activo de transformación de la situación existente. No es ya la perfección como meta final [lo que constituye la finalidad en el vivir], sino el proceso siempre en marcha del perfeccionismo, del refinamiento, del camino hacia la madurez [...]. El crecimiento mismo es el único fin moral (p. 11).

Es gracias a esta educación moral como proceso, que el individuo se orienta hacia la autonomía y la crítica, que se dirige a la acción razonable. Y es precisamente la conjunción entre la educación moral entendida como contenido y 
programa de filosofía para niños como propuesta de educación moral: análisis comparado con otros enfoques de la educación moral

como proceso, la que confiere carácter holístico a la educación moral en la concepción de Lipman:

la educación moral no se limita a ayudar a los niños a saber qué hacer; debe demostrarles cómo hacerlo y darles la oportunidad de practicar el hacer las cosas que pueden elegir hacer en una situación moral dada. Sin esta dimensión del hacer, la educación moral se derrumba. En ninguna (sic.) área es tan importante el lazo entre teoría y práctica como en el área de la educación moral y, sin embargo, en ningún sitio es tan frecuente no tomarlo en cuenta (LIMPAN y al., 1998, p. 253).

Este carácter holístico de la educación moral lipmaniana se manifiesta, al mismo tiempo, al poner en juego no sólo las dimensiones cognitivas del ser humano, sino también las afectivas y las prácticas. En este sentido, el planteamiento de educación moral en Lipman conecta de lleno con el movimiento de Clarificación de valores (Raths, Harmin y Simon, 1966), que considera tres dimensiones en el conocimiento del valor: la cognitiva, la afectiva y la comportamental, ésta última referida directamente a la acción. No resulta extraña esta coincidencia, dado que las dos propuestas de educación moral tienen una misma base pragmática, al alimentarse ambas, la $\mathrm{FpN}$ y la Clarificación de valores, esencialmente de la obra de Dewey (Laprée, 2002). Sin embargo, si bien la coincidencia es notoria en este aspecto, no lo es así en otros. A nuestro parecer, la propuesta de $\mathrm{FpN}$ propone un diálogo de carácter abierto, que no lo es tanto en la metodología encerrada en siete criterios para orientar el debate que propone la Clarificación de valores. Además, en la Clarificación de valores es fundamentalmente el profesor el que provoca el debate con sus preguntas, mientras que en $\mathrm{FpN}$ uno de los roles principales del estudiante en comunidad de investigación es, precisamente, el de formular preguntas que reflejen las propias inquietudes y los propios intereses. Más que de una clarificación de valores, Lipman y al. (1998) hablan de una investigación en valores: "una gran parte de la educación en valores incluye animar a los niños a implicarse en una investigación en valores y ayudarles a hacerla bien" (p. 317). Sin embargo, a pesar de las diferencias, queremos señalar que el enfoque de la Clarificación de valores puede resultar complementario al de $\mathrm{FpN}$ en materia de evaluación: evaluación del desarrollo moral de los estudiantes, así como evaluación de la eficacia de un programa de educación moral. En ambos sentidos, la propuesta de la Clarificación de valores podría ayudar a la evaluación en $\mathrm{FpN}$, aspecto éste menos tratado en el enfoque lipmaniano de educación moral ${ }^{3}$.

Al postular una educación moral holística, Limpan y al. (1998), además de romper con la dicotomía afectivo-cognitivo, rompen también con otra de las

\footnotetext{
3 Para profundizar en este aspecto evaluativo de la propuesta de la Clarificación de valores, invitamos al lector a consultar Laprée (2002).
}

676 childhood \& philosophy, rio de janeiro, v. 14, n. 31, set.-dez. 2018, pp. 671-695 issn 1984-5987 
dicotomías más presentes en educación, la dicotomía hecho (objetivo) - valor (subjetivo):

el resultado de tratar los valores en sí mismos, separados de todo lo demás, es convertirlos en abstracciones muertas o, lo que es incluso peor, involucrar a los niños en discusiones interminables sobre lo que queremos o deseamos, en vez de sobre lo que realmente importa (p. 274).

Para Lipman y al. (Ibid.) las cuestiones son simultáneamente de hecho y de valor y este es el punto principal a tomar en cuenta cuando quiere alcanzarse una coherencia pensamiento-acción, base de la integridad moral y fundamento del enfoque moral de FpN:

Un niño que tiene integridad, es decir, cuyos pensamientos y acciones son coherentes entre sí, se resistirá a realizar una actividad incompatible con el resto de su vida y, de hecho, se escandalizará y disgustará con aquello que está tan distante de su práctica normal. No necesitará que sus padres [o sus profesores] le repitan que no debe decir mentiras, de la misma manera que no será necesario que se le diga que no se corte cuando use el cuchillo (p. 294).

Además, esta coherencia de que hablamos, no se reduce sólo a la del pensamiento y la acción, sino que se extiende a la coherencia entre los diferentes principios morales formulados por un mismo individuo, así como a la coherencia necesaria entre los diferentes comportamientos que una misma persona tiene (Daniel, 1992). La importancia de la coherencia en educación moral entendida de esta manera, nos remite directamente a la relevancia de la lógica en el enfoque de educación moral de FpN. Efectivamente, la coherencia es uno de los elementos en juego en las relaciones lógicas que deben darse en toda educación moral. Otras dos relaciones lógicas fundamentales en educación moral son la relación parte-todo y la relación medio-fin. Si el estudiante entiende que forma parte de una comunidad y que su presencia marca una diferencia sustancial en dicha comunidad (relación parte-todo), así como que es un ser en relación y que, por tanto, su crecimiento personal repercute en el crecimiento de la comunidad (relación medio-fin), es que podremos hablar de un crecimiento moral como crecimiento del sentido moral de la existencia gracias a la experiencia significativa: "la búsqueda de sentido [moral] equivale a la búsqueda de la relación entre una cosa y su contexto, entre la parte y el todo, entre el medio y el fin" (Ibid., p.64). La importancia de entender el ser como ser en comunidad de relaciones sociales, según la concepción lipmaniana, nos remite directamente a la relevancia de la comunidad de investigación en la educación moral. 
programa de filosofía para niños como propuesta de educación moral: análisis comparado con otros enfoques de la educación moral

\section{fpn y los enfoques éticos centrados en la intersubjetividad: la comunidad de investigación ética en el corazón de la educación moral}

Ya hemos señalado con anterioridad que la propuesta del programa de FpN para la educación moral es una propuesta de investigación en valores, investigación presidida por el descubrimiento y entendida como "la constancia en la exploración auto-correctiva de temas [con contenido moral] que se perciben al mismo tiempo como algo problemático e importante" (Lipman y al., p. 40). En este enfoque de educación moral como investigación, la observación, la demostración y la experimentación ocupan un lugar análogo al que ocupan en el resto de las ciencias, confiriendo al conocimiento moral resultante un carácter de conocimiento científico (Miranda, 1991). Pero, no sólo el aspecto de la investigación es crucial a la hora de la práctica de $\mathrm{FpN}$, sino también el hecho de que esta investigación se lleva a cabo en comunidad, en contraposición a un aprendizaje en solitario que priva al niño:

de la experiencia vital de la cooperación intelectual, de construir sobre las ideas de los demás, de apreciar la novedad de las interpretaciones ajenas, de defender las propias ideas cuando son criticadas, de disfrutar de la solidaridad de los otros y de darse cuenta de su integridad intelectual cuando revisa el propio punto de vista a la luz de nuevas opiniones (LIPMAN y al., p.107).

Condiciones todas necesarias para que se dé una educación moral, basada en la disponibilidad a la razón, en el respeto mutuo y en la ausencia de adoctrinamiento, y tendente al desarrollo de la autonomía moral, gracias a la participación en una comunidad de investigación centrada en el diálogo filosófico. La transformación del aula en comunidad de investigación ética, implica la transformación del aula en una micro-sociedad democrática donde los niños pueden vivir experiencias que ponen en juego todos los ingredientes de una vida democrática facilitadora del real desarrollo de la naturaleza humana (Dewey, 1922/1964). Para el autor, sólo en las sociedades democráticas puede desarrollarse plenamente la naturaleza humana y sólo a través del desarrollo de la naturaleza humana puede desarrollarse el yo moral, componente indisociable de dicha naturaleza humana. Por tanto, una escuela será moral, y por ende legítima y deseable, en tanto que potencie ese desarrollo de la naturaleza humana en contexto democrático. En la propuesta de $\mathrm{FpN}$, este contexto democrático queda asegurado por el funcionamiento, centrado en el diálogo, en comunidad de investigación. Gracias a la progresión del diálogo filosófico en comunidad de investigación ética, el niño es capaz de cuestionar sus propios argumentos morales para autocorregirlos a la luz de lo que piensan los demás miembros de la comunidad. De esta manera, la comunidad de investigación ética se convierte en piedra angular de la propuesta de educación moral de $\mathrm{FpN}$, como espacio de actualización 
permanente del discurso ético, tanto individual como grupalmente. Para que esto sea así, la comunidad de investigación ética debe ser, según Sharp (1990), un espacio de escucha activa de las ideas de los otros, un espacio de libre construcción de ideas y juicios a partir del ejercicio del propio pensamiento y a la luz de las ideas de los demás. Debe ser, al mismo tiempo, un espacio de coherencia argumental y de coherencia pensamiento-acción. La comunidad de investigación ética es, en definitiva, un espacio donde, gracias a la propuesta de ideas, unas veces complementarias y otras veces alternativas, se favorecen la autocorrección y el desarrollo de la autonomía moral. El diálogo es el elemento esencial en juego para que esto se produzca:

hablando con los demás es como llega uno a ser realmente persona. Hablando con los demás es como el mundo se compromete con la realidad [...] Lenguaje y pensamiento son actividades solapadas. Decir las propias ideas a los compañeros de clase es crear y expresar nuestro propio pensamiento, $\mathrm{y}$, en cierto sentido, crearnos a nosotros mismos (Ibid, p.11).

En efecto, de acuerdo con Sharp (Ibid.), el proceso de hacerse persona en todos los sentidos, y también en el moral, es un proceso que se da en comunidad y gracias al diálogo. En esto, el planteamiento de $\mathrm{FpN}$ coincide con cinco de los principales planteamientos contemporáneos de educación moral: el de la solicitud, el narrativo, el discursivo, el reconstructivo y el de la razón cordial.

\section{fpn y ética de la solicitud: el otro en el corazón de la educación moral}

Desde la ética de la solicitud (Gilligan, 1982; Noodings, 1993), que coloca en el centro del interés ético el aspecto relacional y de cuidado del Otro, se hace una propuesta de educación moral en torno a cuatro elementos principales: la práctica de la cooperación y de la interrelación; el rol esencial de los modelos en la educación moral; la confirmación como modo de afirmación del Otro y de compromiso con su experiencia de vida; y el diálogo, que pone en juego la receptividad y la racionalidad (Gendron, 2002). Tomado en este sentido, el diálogo en el enfoque de la solicitud para la educación moral cumple un rol similar al que le es concedido en la aproximación de FpN. Sin embargo, según Gendron (Ibid.), es en el modo de dirigir el diálogo donde estriba la diferencia. Según la autora, mientras que en $\mathrm{FpN}$, el diálogo queda circunscrito a un método de lecturarecolección de ideas, en el enfoque de la solicitud el diálogo tiene un carácter mucho más abierto. Sin embargo, desde nuestro punto de vista, la conclusión de Gendron (Ibid.) es fruto de una lectura errónea de la propuesta de FpN. En efecto, el método de $\mathrm{FpN}$, de lectura y recolección de preguntas que los propios niños se formulan (más que recolección de sus ideas), es, más bien, una manera de abrir el diálogo a la comunidad de investigación y de centrarlo en los intereses del niño. El niño, al intervenir en todo el proceso de diálogo, se convierte así en protagonista directo del curso que toma dicho diálogo en la comunidad de investigación. Es por 
programa de filosofía para niños como propuesta de educación moral: análisis comparado con otros enfoques de la educación moral

esto que, contrariamente a la afirmación de Gendron (Ibid.), el diálogo en comunidad de investigación ética posee un carácter mucho más abierto que en el enfoque de la solicitud, por estar menos condicionado por los intereses y consideraciones previas del profesor y más situado en los de cada niño y niña participante en la comunidad.

Efectivamente, el enfoque de educación moral de la solicitud, postulado esencialmente por Noodings (1993), puede resultar excesivamente directivo, al concebir al profesor como ser que cuida de unos alumnos menos equipados que él en materia moral. Este enfoque del cuidado queda superado cuando se entiende la comunidad de investigación ética como un espacio donde todos cuidan de todos, profesor-estudiantes y viceversa, así como los estudiantes entre sí. Es sólo en esta relación de igualdad entre todos los miembros de la comunidad de investigación ética, que el verdadero cuidado, y por ende el verdadero desarrollo moral, pueden darse. Es sólo desde ahí que la experiencia del niño cobra sentido en el presente y no sólo como medio para alcanzar el mismo estadio de ser cuidante que sustenta el profesor. En este sentido, el enfoque de la solicitud, al quedar encerrado en una visión rígida y jerárquica del desarrollo moral, pecaría de lo mismo que la teoría del desarrollo moral de Kohlberg al no valorar la realidad moral del niño en su justa medida. Superar esta idea, reconociendo la categoría moral de los niños en condiciones de igualdad con los adultos, es uno de los principios básicos de $\mathrm{FpN}$, punto de arranque de toda su propuesta educativa.

Esta relación de desigualdad profesor-estudiante se traslada también al rol que los modelos cumplen en la educación moral a la luz de la ética de la solicitud. Desde esta aproximación, el papel del docente como modelo moral es fundamental e inalienable: "en el enfoque de la solicitud, la formulación de testimonios personales por parte del profesor o la profesora es considerado moralmente obligatorio, en virtud de un objetivo particular, intentar mantener o acrecentar el ideal ético de los estudiantes" (Gendron, 2002, p.74, traducción libre). Sin embargo, esta concepción reductora de la importancia de modelos en educación moral, viene superada por el enfoque de $\mathrm{FpN}$, al proponerse como modelos morales de relevancia, no sólo a los profesores, sino, además, al grupo de iguales participando en comunidad de investigación ética, así como a los personajes protagonistas de las novelas que se utilizan como punto de partida de la discusión (niños de la misma edad que aquellos a quienes va dirigida la novela), modelos igualmente de pensamiento y actuación moral. Esta importancia de los personajes de las novelas de Lipman como modelos de referencia para los niños, hace coincidir el enfoque de $\mathrm{FpN}$ con el enfoque narrativo, que subraya la relevancia de la literatura en la educación moral.

\section{fpn y ética narrativa: la narración en el corazón de la educación moral}


El principio esencial del enfoque narrativo de la educación moral expone que el sentido moral de un individuo es el fruto de la interacción entre la propia historia y las historias de otros (Morris y Caron, 2002). En este sentido, el fin de la educación moral sería la construcción permanente y la apropiación de la historia personal a partir del diálogo con las historias de los demás. Así, desde el enfoque narrativo, la construcción permanente de esta historia personal necesita de la integridad del ser humano, es decir, requiere de su pasado, se sitúa en su presente y se proyecta hacía un futuro. Lo que en palabras de Dewey (1922/1964) podría traducirse como que la historia personal es la construcción permanente de la experiencia, experiencia entendida como lugar de encuentro del pasado con el presente y de ambos con el porvenir. De este modo, la propuesta del enfoque narrativo de la educación moral coincidiría en sus postulados de base con el pensamiento deweyano y, por ende, con los fundamentos de la educación moral del programa de $\mathrm{FpN}$, sustentados en dicho pensamiento.

Pero, si en algo coinciden los dos planteamientos, además de en sus postulados de base, es en la importancia concedida a la narrativa, a las historias personales y de otros, a la hora de hablar de educación moral. La diferencia fundamental estribaría, sin embargo, en el modo de elaborar y emplear dicha narración: mientras que en $\mathrm{FpN}$, la narración ocupa un lugar principal en el momento de la lectura comunitaria de la novela, en el modelo de educación moral del enfoque narrativo se propone que el estudiante construya su propia historia a través de la entrevista personal con el profesor, la redacción de un diario personal o la dramatización individual en clase; mientras que, en la propuesta lipmaniana, lo central es la narración de la vida de los personajes de las novelas creadas por el autor para practicar el programa, en el enfoque narrativo lo es la narración de la historia personal del estudiante. Sin querer decir por ello que cada una de las propuestas excluya a la otra, ya que el enfoque narrativo también recurre a vida de personajes literarios, tanto como la presencia de la experiencia personal forma parte de la concepción de Lipman. Es tan sólo una cuestión de acento la que marca la diferencia.

Sin embargo, según Bouchard (2000), la propuesta de educación moral del enfoque narrativo no es realista (por falta de tiempo para escuchar seriamente la historia de cada estudiante de la clase), ni aconsejable por el riesgo que implica de que la actividad se convierta en una forma de terapia y se aleje de unos fines que deberían ser puramente educativos, preocupación ésta también compartida en la comunidad educativa de FpN. Efectivamente, son evidentes los riesgos de que el diálogo en comunidad de investigación derive en asuntos personales traídos por los niños y las niñas a la escena comunitaria, mucho más cuando se trata de temas de corte moral, donde entran en juego de lleno los valores, las vivencias cotidianas y las creencias personales. De ahí que en FpN, afín de evitar personalizar excesivamente el diálogo, se promueva el diálogo de carácter filosófico en comunidad de investigación. En este diálogo acumulativo, las ideas de uno se van construyendo sobre las de los demás: 
programa de filosofía para niños como propuesta de educación moral: análisis comparado con otros enfoques de la educación moral

Una mera discusión es lineal y episódica, como una novela picaresca mediocre en la cual hay una serie de episodios colocados uno después del otro, pero a partir de los cuales nunca se construye nada. Por otro lado, una buena discusión es acumulativa; cada contribución es, en efecto, una línea de fuerza o un vector que converge con las otras y es orquestada con ellas. El que haya completo acuerdo o desacuerdo al cierre del episodio tiene poca importancia; lo que importa es que las contribuciones de cada participante se relacionan y se refuerzan unas con otras conforme cada participante aprende a partir de lo que otros han dicho (LIMPAN y al., 1998, p. 206).

Para favorecer este desarrollo acumulativo del pensamiento, en las discusiones filosóficas las ideas deben estar al servicio del avance de la investigación comunitaria y no al servicio de intereses personales ajenos a dicha investigación. Esto no quiere decir que el niño no pueda hacer referencia a su propia experiencia vital. Más bien, según hemos visto, dicha experiencia se sitúa en el centro del diálogo en comunidad de investigación. Pero lo que sí debe evitarse a toda costa, es que la narración de una experiencia adquiera tintes de consulta terapéutica, ya que no es el fin de la comunidad tratar directamente de la resolución de conflictos personales, sino favorecer el progreso de la investigación conjunta. El espacio para la resolución de estos conflictos queda fuera de los límites de la comunidad de investigación y requiere el concurso de otros servicios, como, por ejemplo, los de apoyo psicológico a estudiantes. De ahí que, considerando el enfoque narrativo en su conjunto, la propuesta de $\mathrm{FpN}$ se aproxime más a la de Bouchard (2000), centrada también en el diálogo, que a otras propuestas del enfoque narrativo que ponen el acento en la narración individualizada de la historia personal.

Otras líneas de pensamiento de la educación moral desde un enfoque narrativo (Nussbaum, 1990 y 1995; Johnson, 1993, en Morris y Caron, 2002) resaltan, al igual que la propuesta de $\mathrm{FpN}$, el papel central que juega la imaginación moral en el crecimiento moral del individuo y las posibilidades de la literatura para el desarrollo de dicha imaginación moral. Efectivamente, Limpan y al. (1998) señalan como herramienta importante de la educación moral, el desarrollo de la imaginación moral, como amplitud de "la capacidad para manejar imaginativa y creativamente situaciones que, de otro modo, resultarían difíciles y confusas" (p. 279). Este desarrollo de la imaginación moral no excluye el concurso de la razón moral en ninguno de los tres enfoques mencionados, si bien pensamos que, mientras que en FpN la balanza se inclina del lado de la razón, en el caso de los enfoques de Nussbaum (Ibid.) y Johnson (Ibid.) lo hace del lado de la imaginación moral. En cualquier caso, y tal como señalan Morris y Caron (2002), el objetivo común es pensar con historias más que pensar sobre historias, afín de que 
el pensamiento sirva a la construcción de la propia historia personal como meta de la educación moral.

Tras haber analizado la propuesta ética lipmaniana a la luz de los enfoques narrativo y de la solicitud, pasamos a hacer lo mismo con otro enfoque de educación moral también centrado en el diálogo como elemento principal de crecimiento moral: el enfoque de la ética discursiva de Apel y Dussel (2005) y Habermas (2000 y 2008), ampliamente reconocido en la actualidad.

\section{fpn y ética del discurso: el diálogo en el corazón de la educación moral}

La ética del discurso es una ética de corte racionalista marcada por la intercomprensión como elemento clave a la hora de hallar normas con pretensión de validez universal (Cortina, 1986). Su finalidad es la búsqueda del consenso racional gracias a la constitución de "comunidades ideales de argumentación" (Apel y Dussel, 2005), donde todos los participantes pueden expresarse en "situaciones ideales de habla" (Habermas, 2000 y 2008). Así pues, la validez universal de la norma vendrá avalada por contar en el proceso de su elaboración y aceptación con todos aquellos a quien dicha norma concierne. En este sentido, todo ser humano es un interlocutor válido. Los intereses humanos que fundamenten un consenso racional, serán, pues, los intereses con carácter universal y generalizables, no los intereses individuales que no toman en cuenta el sentir de todos.

Desde este planteamiento ético, Cortina (Ibid.) sugiere que: son rechazables todas las éticas que, por su concepción del hombre, desautoricen a algunos de ellos como interlocutores válidos; los contenidos de la moral vendrán constituidos por los intereses y convicciones generalizables; la identidad humana se construye a partir de contenidos decididos consensuadamente, a través de diálogos en los que se consideren los intereses de todos; la identidad es un proyecto a lograr a través de contenidos en los que los hombres nos reconozcamos; la determinación de la moral es progresiva, por lo que todo consenso fáctico es revisable a través del largo camino hacia la comunidad de diálogo ideal.

Estos aspectos señalados por Cortina tienen puntos de encuentro con el planteamiento moral de FpN. En primer lugar, en la comunidad de investigación ética, los participantes tienen el mismo estatus que en las comunidades ideales de habla propuestas desde la ética del discurso, es decir, idéntica validez como interlocutores. En segundo lugar, ambas propuestas se centran en el uso del diálogo y conceden una importancia central a la argumentación racional elaborada gracias al uso de dicho diálogo en comunidad. Por último, desde ambos enfoques se defiende que la identidad moral humana no se adquiere de una vez por todas, sino que, más bien, está en permanente construcción.

Sin embargo, encontramos también acentuadas discordancias entre la propuesta de educación moral de $\mathrm{FpN}$ y la que se puede inferir desde la aproximación de la ética del discurso. Por un lado, la importancia concedida a la razón no es la misma. Mientras que la ética del discurso apela a la razón como elemento decisivo en el logro del consenso en comunidad, desde FpN se entiende 
programa de filosofía para niños como propuesta de educación moral: análisis comparado con otros enfoques de la educación moral

que el concurso de las habilidades afectivas del ser humano es igualmente decisivo. Por otro lado, mientras que la ética del discurso tiene una pretensión de universalidad en su propuesta, al proponer como meta el consenso de todos para la elaboración de principios morales generalizables, no es esa la pretensión de la propuesta de FpN. Partiendo del principio de relación indisoluble entre la parte y el todo, desde $\mathrm{FpN}$ se pone el acento en el desarrollo moral del individuo, entendiendo a la vez que dicho desarrollo moral individual tiene consecuencias positivas sobre la comunidad de investigación ética y viceversa. Entendemos, sin embargo, que cuando Cortina (Ibid.) dice que la identidad humana, "se construye a partir de contenidos decididos consensuadamente" (Ibid.), de lo que habla es de una ética de mínimos exigibles a todos, según criterios de justicia, más que de una pretendida universalidad de valores humanos de manera general. Efectivamente, para la autora, aunque no es posible exigir a todos los hombres que se guíen por unos criterios de felicidad, sí que es exigible a todos los hombres hacerlo según unas orientaciones de justicia:

la clave de nuestro sentir acerca de la justicia no radica en el conocimiento intuitivo de principios materiales ni en el deseo de felicidad, sino en el concepto de persona moral que hemos ido asimilando al hilo de una larga tradición, en el que nos reconocemos, y al que ya no estamos dispuestos a renunciar. A partir de este concepto, en torno al cual existe acuerdo implícito en las sociedades democráticas, delinearemos los rasgos de los principios de justicia desde los que enjuiciaremos cualquier norma o ley (Ibid., p. 188).

En este sentido, Cortina (Ibid.) - como Lipman y otros autores principales al hablar de educación en valores, como Reboul (1992), Gervilla (1998), Escámez (1998) o Fullat (2004) - , al reconocer dentro del vasto mundo de los valores un grupo más reducido común a toda la humanidad, trata de romper con objetivismos y relativismos en el ámbito moral. Sin embargo, podría hacerse a Cortina (Ibid.) la misma crítica que se hace a Kohlberg (1984) de manera generalizada en el panorama actual de la educación moral al conceder un peso excesivo, y casi exclusivo, al valor de la justicia en el momento de determinar los estadios del desarrollo moral (o la determinación de normas éticas universales, en el caso de Cortina, Apel, Dussel y Habermas). La justicia acapararía así el panorama axiológico común a la humanidad, frente a otros valores, tales como la empatía, central en el pensamiento tanto de Dewey como de Lipman, así como en el enfoque de la solicitud que hemos analizado con anterioridad. Sin embargo, un análisis detallado tanto de la obra completa de Kohlberg como de Cortina, nos revela que ambos autores parecen darse cuenta de esta y otras carencias, según progresan en la elaboración de sus propuestas éticas. En lo que concierne a Kohlberg y sus seguidores, llegaron a reconocer que la reducción de la educación moral al trabajo con dilemas morales, no era suficiente. Así, Hers, Reimer y Paolitto (2002), señalan 
la importancia de trabajar no sólo sobre casos hipotéticos, sino también reales, además de la relevancia de trabajar la educación moral en comunidad, ya que el pensamiento compartido es el que favorece la elaboración del propio pensamiento.

De esta manera, los autores reconocen que otros valores, relacionados con la vida comunitaria, como la empatía y la solidaridad, por ejemplo, son tan esenciales como la justicia a la hora de evaluar dicho desarrollo moral. Además, Hers y al. (Ibid.), reconocen las oportunidades que ofrece la literatura universal al rescatar historias que presentan conflictos de valores al estilo de los dilemas ideados por Kohlberg, aproximándose también de esta manera a la propuesta de FpN, así como a la del enfoque narrativo de educación moral. En cuanto a la obra de Cortina, el acercamiento a la postura de $\mathrm{FpN}$ y de la solicitud queda patente al analizar la progresión de su obra, tal y como veremos a continuación.

\section{fpn y las éticas reconstructiva y de la razón cordial: el corazón en el corazón de la educación moral}

A lo largo de la extensa reflexión de Cortina sobre la cuestión ética, desde la Ética mínima de 1986, hasta la Ética de la razón cordial de 2007, pasando por la Ética sin moral (1990), La ética de la sociedad civil (1994), la Ética civil y religión (1995) y la ética de los Ciudadanos del mundo (1997), entre otros títulos, hallamos el pensamiento kantiano como telón de fondo. Sin embargo, en Cortina ese pensamiento kantiano, a menudo reducido a su vertiente racional, cobra nuevas dimensiones gracias a la lectura personal que hace la autora de Kant (1724-1804), cuando dice:

A pesar de lo que se ha dicho y escrito sobre la ética kantiana, lo bien cierto es que en sus obras nuestro bagaje moral se delinea como mucho más amplio que el de la sola razón. El mismo hecho de percatarse de que la razón da leyes no es una acción de la razón, sino de la sensibilidad, de ese elemento constitutivo del ser racional al que llamamos "conciencia moral". (CORTINA, 2007, p. 120)

En este mismo sentido la autora señala que al hablar de justicia es preciso contar con las razones de la razón tanto como con las razones del corazón, no pudiéndose reducir la comunicación al discurso lógico (Cortina, Ibid.). De este modo y sin abandonar en ningún momento el telón de fondo kantiano, Cortina (Ibid.) defiende la importancia de una razón cordial en moral (y, por ende, también en educación moral), definida como una razón que necesita del corazón (de ahí que sea cordial). Recíprocamente, los proyectos del corazón se conocen e interpretan a la luz de la razón. En este sentido, el planteamiento de la razón cordial en Cortina (Ibid.), daría crédito al mismo tiempo a los planteamientos de Kohlberg y de la ética del discurso (ambos centrados en la argumentación racional sobre la justicia), así como a la propuesta de la solicitud, que sitúa en el centro de la escena moral a la compasión y al cuidado, valores éstos más cercanos al corazón que a la razón: "alcanzar la madurez moral no consiste sólo en llegar a ser justo, 
programa de filosofía para niños como propuesta de educación moral: análisis comparado con otros enfoques de la educación moral

sino también en lograr ser compasivo y capaz de responsabilizarse de aquellos que nos están encomendados" (Cortina, Ibid., p. 150). En este sentido, la autora se aproxima al enfoque moral reconstructivo de Ferry (1991, en Bouchard, 2002) donde se articulan dos corrientes éticas dominantes: la corriente que estima que gracias a la narración se construye la identidad y la ética adviene (línea de pensamiento de Ricoeur (2005), MacIntyre (1981, en Bouchard, Ibid.) y Bouchard (2000), entre otros); y la corriente que estima, al hilo de Kant (1781/2005) y Rawls (2002), que la razón pública es la que permite el advenimiento de la ética (línea de pensamiento de Apel y Dussel (2005) y Habermas (2000 y 2008). Esta conciliación de corrientes de pensamiento en Filosofía moral, se concretiza en el pensamiento de Ferry (Ibid.), cuando señala que no es el reconocimiento de los argumentos, sino el de las personas que los formulan, lo que está en el centro de la ética. De esta manera, en su formulación, Ferry (Ibid.) reúne al ser humano con su propio argumento, a la razón con el afecto.

Por su parte, la propuesta de FpN se situaría muy cerca de estas aproximaciones de Ferry (Ibid.) y Cortina (2007), en cuanto que supone también una ruptura de la dicotomía afectivo-cognitivo, razón-corazón. Otros puntos de coincidencia entre estas tres concepciones de la moral en general y de la educación moral en concreto, vienen de la mano de la importancia central concedida al diálogo, de la necesidad de coherencia pensamiento-acción y de la irrenunciable condición intersubjetiva del crecimiento moral personal, idea ampliamente sostenida en la historia del pensamiento ético desde Hegel $(1807 / 2006)$ hasta autores contemporáneos como Levinas (2007), Horkheimer (2000), Puig Rovira (2003), Ricoeur (2005) y Bauman (2006). Éste último nos propone una ética postmoderna que devuelve al Otro a la escena moral, tras haber sido borrado de ella en la comprensión ética de la modernidad:

en un mundo construido únicamente con reglas codificables, el Otro permanecía en el exterior de este yo como una presencia misteriosa pero, ante todo, confusamente ambivalente [...] En la ética moderna, el Otro era la contradicción encarnada y el más temible obstáculo para que el yo lograra la plenitud [...] la ética postmoderna readmitiría al Otro como vecino -como aquel que está cerca del cuerpo y de la mente- en lo más profundo del yo moral, en su retorno del erial de los intereses calculados al que había sido exiliado [...] volvería a forjar al Otro como el personaje central del proceso mediante el cual el yo moral llegaría a serlo (Bauman, 2005, p. 98).

Y, en consonancia con el autor, Cortina (2007) señala que es este vínculo intersubjetivo el que obliga internamente al sujeto moral a actuar como tal. Al reconocer al Otro como interlocutor válido, el sujeto moral queda vinculado al Otro, pero no por una obligación externa, sino por una obligación auto-impuesta, dado que, al reconocer al Otro, el Otro pasa a ser parte constituyente del sujeto 
moral. En el caso de FpN este reconocimiento y cuidado del Otro se concretiza en la comunidad de investigación ética, a través, fundamentalmente, de lo que Lipman denomina el pensamiento cuidante.

\section{fpn: el pensamiento cuidante en el corazón de la educación moral}

El pensamiento cuidante está presente en la propuesta de Lipman como un complemento necesario a la diada pensamiento crítico - pensamiento creativo, entendidos ambos como elementos del pensamiento de orden superior. De esta manera, el pensamiento cuidante se sitúa en la escena del pensamiento humano para favorecer la formación de la persona integral en todas sus dimensiones y para ampliar, concretamente, el dominio de lo moral (Lago, 2006).

Cabe entonces preguntarse, qué es lo que añade el pensamiento cuidante a un pensamiento de orden superior constituido por sus dos dimensiones crítica y creativa. Y ahí es donde Lipman (2003) responde que esta nueva vertiente del pensamiento de orden superior es la que permite al sujeto moral preocuparse, precisamente, por el Otro. Sin este tercer componente del pensamiento de orden superior, éste queda incompleto: en la distinción entre pensamiento ordinario y pensamiento complejo debemos mantener el enfoque tripartito enfocado a los ideales de la verdad, el sentido y el valor. Mientras que el pensamiento crítico se comprometería con la búsqueda de la verdad, el creativo lo haría con la búsqueda del sentido y el cuidante con la del valor (Lipman, 1995).

Así, Lipman (1991), al reconocer no sólo el componente afectivo del cuidado, sino también el cognitivo, define el cuidado como categoría de pensamiento. De esta manera, el autor rompe de nuevo con las dicotomías, en este caso con la dicotomía cognitivo-afectivo, ruptura de dicotomías que recorre, al igual que en el caso de Dewey, todo su pensamiento (Pérez de Tudela, 1988).

Además, según Lago (2004), la concepción lipmaniana del pensamiento cuidante no se reduce a su vertiente de pensamiento centrado en valores, sino que es también un pensamiento valorativo en sí, esto es, capaz de emitir "un juicio sobre la persona, sus acciones y conductas y sobre el mundo que le rodea, es un pensamiento comprometido con la persona y el entorno" (Ibid., p. 109). Es decir, el pensamiento cuidante es un pensamiento no sólo de ideas sino de acción, un pensamiento a partir del cual el sujeto moral es capaz no sólo de percibir el mundo, sino de imaginar también el mundo en el que le gustaría vivir, de imaginar tanto lo que el mundo podría ser como lo que el mundo debería ser. De esta manera, el pensador cuidante se compromete con la construcción de un mundo más justo, en relación con el Otro entendido en el sentido amplio de toda la humanidad, así como con el medio ambiente en su concepción más vasta de planeta Tierra. En este sentido, el pensamiento cuidante orienta las metas, los ideales y las acciones del individuo. 
programa de filosofía para niños como propuesta de educación moral: análisis comparado con otros enfoques de la educación moral

conclusiones preliminares: fpn, programa integrador y original para la educación moral

En esta sección de nuestro trabajo, hemos presentado el programa $\mathrm{FpN}$ en su vertiente ética, resaltando sus elementos principales al hilo de la comparación con otros enfoques contemporáneos de la educación moral. Este análisis comparado, nos ha permitido comprobar la validez y actualidad de la FpN en el contexto de educación moral.

Efectivamente, nos ha permitido, en primer lugar, verificar la validez de la comunidad de investigación ética como espacio óptimo de educación moral. En segundo lugar, nuestro análisis demuestra la pertinencia de una propuesta basada en el diálogo filosófico, como la de $\mathrm{FpN}$, cuando se trata de educar el sujeto moral. En tercer lugar, el programa de $\mathrm{FpN}$, en su planteamiento holístico, que parte de una concepción integral de la persona y que tiende a la ruptura de dicotomías, especialmente la afectivo-cognitiva en materia moral, se corresponde con las principales propuestas de educación moral de hoy en día. En cuarto lugar, y también aproximando la propuesta ética de $\mathrm{FpN}$ a otros enfoques contemporáneos, podemos afirmar la necesidad de ligar el pensamiento a la acción en educación moral. En quinto lugar, nuestro estudio revela la absoluta pertinencia de considerar la narración como elemento fundamental del desarrollo moral, tal como sucede en la propuesta de FpN. Finalmente, también se ha mostrado que la propuesta de $\mathrm{FpN}$ para la educación moral, no es solamente integradora (por recoger elementos esenciales reclamados por otros enfoques contemporáneos), sino original, al asociar el desarrollo moral con el desarrollo de un pensamiento de orden superior que requiere el concurso de tres tipos de pensamiento: el crítico, el creativo y el cuidante. Todos estos aspectos del enfoque de $\mathrm{FpN}$, le confieren absoluta validez como programa de educación moral. Cabe ahora preguntarnos por los límites de esta propuesta tan integradora como original. De estos límites trataremos en la sección siguiente.

\section{crítica del programa de fpn como programa de educación moral}

Según Daniel (1992 y 2008), la crítica al programa de FpN como programa de educación moral gira en torno a algunas cuestiones principales: el impacto del diálogo sobre la conducta moral de los niños; la influencia del trabajo en comunidad de investigación ética sobre la actuación del niño en contextos extraescolares; las posibilidades de una educación moral holística como la propuesta desde el programa; $\mathrm{y}$, por último, los riesgos de propiciar con esta práctica el relativismo moral.

Respecto al impacto del diálogo sobre el desarrollo moral de los niños, Daniel (Ibid.), subraya que en la historia del pensamiento humano este impacto ha sido negado con frecuencia. Así, para Platón (427 a.C/428 a.C - 347 a.C.), por 
ejemplo, toda persona, antes de embarcarse en el diálogo ético, debe conocer los principios fundamentales de la moral. Desde este punto de vista, el niño (y también el joven estudiante) quedaría excluido del diálogo moral hasta no haberse apropiado en la edad adulta de dichos principios morales.

Esta misma línea de pensamiento la encontramos muy presente en nuestros días gracias a la gran influencia de Piaget en el ámbito de la educación. Para Piaget, este diálogo ético no puede darse de manera real en el niño, dado el carácter egocéntrico de la naturaleza infantil, lo cual le incapacita para reconocer verdaderamente al Otro y, por ende, para dialogar con él. Sin embargo, según señala Daniel (Ibid.), el niño es capaz de apertura al Otro, de autonomía, de razonamiento lógico y de auto-crítica, lo cual le habilitaría adecuadamente para el diálogo ético, tal y como se propone en FpN.

En realidad, para Daniel (Ibid.), y para nosotros de acuerdo con ella, la reserva fundamental frente al diálogo que provoca el desarrollo moral radica en el hecho de cómo asegurar la calidad de dicho diálogo, para que sea efectivamente filosófico y de carácter ético, y no interpretado y practicado por el niño como una manera más de acumular conocimientos. En este sentido, creemos que la solución pasa por una seria formación del personal docente que practica FpN. Esta será la mejor manera de asegurar la calidad de dicho diálogo. En cualquier caso, según Daniel (Ibid.), es más la comunidad de investigación ética que el intercambio verbal que en ella se da, la que posee el verdadero valor educativo moral. Esto nos conduce al segundo foco de críticas de la apuesta de educación moral de FpN. ¿Hasta qué punto el trabajo realizado en comunidad de investigación ética puede influir el comportamiento del niño en contexto extra-escolar? En este sentido, Daniel (Ibid.) apunta al hecho de que el ambiente propiciado en la comunidad de investigación ética, y los valores de cooperación y empatía (o cordialidad) que rigen su funcionamiento, no son necesariamente acordes con los presentes en la sociedad en general, ni, concretamente, en la práctica educativa dominante. Este hecho dificulta enormemente la puesta en práctica de la $\mathrm{FpN}$, al no estar en consonancia con los patrones y valores educativos priorizados por la sociedad. Además, es cuestionable la repercusión que podría tener la práctica de la $\mathrm{FpN}$ en comunidad de investigación ética, dada la influencia incomparablemente mayor del ambiente social general, fundamentalmente del ambiente familiar.

Sin embargo, las dificultades de la puesta en práctica del programa de FpN, no le restan validez. Más bien al contrario, estas dificultades resaltan su valía, no sólo como programa educativo, sino como programa transformador. En efecto, a nuestros ojos, la FpN es una propuesta "difícil" de educación moral; "difícil" por ser diferente, compleja y transformadora. De ahí, precisamente, su necesidad, ya que la $\mathrm{FpN}$ aporta elementos imprescindibles pero ausentes en la práctica educativa moral dominante, tales como, por ejemplo, la comprensión de la educación moral como proceso de investigación ética conjunta basado en el diálogo, o la importancia concedida a la narración en la educación moral. Estos elementos coinciden con los subrayados por otras corrientes contemporáneas de educación moral. Lo que marca la diferencia de la FpN con estas otras corrientes, 
programa de filosofía para niños como propuesta de educación moral: análisis comparado con otros enfoques de la educación moral

tales como la de la solicitud o la discursiva, es su carácter integrador, que emana de una concepción holística de la educación moral. Esta perspectiva holística rompe con dicotomías tradicionalmente presentes en la educación moral (afectivocognitiva, hecho-valor, parte-todo, medio-fin), a la vez que reúne elementos compatibles de diferentes enfoques, dotando de una riqueza única al programa, cuya contraparte viene definida por las dificultades de la puesta en práctica de una propuesta que encierra tanta complejidad. Es por eso, que Daniel (Ibid.) se cuestiona sobre las posibilidades de aplicación de este planteamiento holístico de la educación moral en $\mathrm{FpN}$, tercer foco de críticas a la propuesta lipmaniana.

Por último, el cuarto foco de críticas señalado por Daniel (Ibid.), se refiere al riesgo de propiciar el relativismo moral con la práctica de FpN. Para la autora, este peligro proviene del hecho de ser la FpN un programa centrado en la discusión ética entre iguales y no en un dictado de principios y actitudes que configuran a todo sujeto moral. Este foco de críticas provendría, fundamentalmente, del enfoque de la educación moral como educación del carácter, de Kilpatrick (1992, en Roy, 2002) y Lickona (1991, en Ibid.), entre otros. Esta aproximación de la educación moral, en la misma línea señalada por Platón anteriormente, promueve la existencia de unos valores universales y absolutos, que deben ser transmitidos a los niños antes de cualquier propuesta de diálogo o clarificación. De este modo, la escuela impone a los niños los valores elegidos por la sociedad. Desde este punto de vista, toda práctica de educación moral que se base en el diálogo, como la de FpN, es relativista y atenta contra el principio básico de universalidad de valores y de escuela como institución encargada de la transmisión de las normas y virtudes que imperan en la sociedad.

Sin embargo, compartiendo los riesgos señalados por Daniel (1992 y 2008), no compartimos en absoluto la postura del enfoque de la educación del carácter. Creemos en las posibilidades del diálogo en comunidad, principio esencial de la educación moral. Y entendemos que, para que este diálogo en comunidad favorezca de manera efectiva el crecimiento moral, debe asegurarse su carácter tanto filosófico como ético, lo cual nos envía directamente, una vez más, a la necesidad de una formación exhaustiva del personal docente que practica la FpN. A nuestro parecer, de acuerdo con Tozzi (2008), sólo una formación de calidad y continua, puede garantizar una práctica de $\mathrm{FpN}$ conforme a los postulados expuestos en la propuesta teórica lipmaniana. Sólo dicha formación puede hacer de la propuesta teórica una realidad práctica.

Además de estos cuatro focos de crítica, señalados por Daniel (Ibid.) para la FpN como propuesta de educación moral, nosotros añadimos dos más. El primero se refiere a la carencia de estudios científicos centrados en las posibilidades del programa en su vertiente moral. Según Mortier (2008), la evaluación del programa de FpN se ha abordado con dos perspectivas. Por un lado, tomando el programa de manera general, a través de la evaluación del desarrollo de las habilidades de pensamiento, como en el caso del estudio de García Moriyón (2002), de Lim (1995, 
en Mortier, Ibíd.) y de Sprod (1997, dans Ibid.). Por otro lado, centrándose en aspectos concretos del programa, como el desarrollo de la autoestima (Sasseville, 1994; Lago, 1990, en Mortier, Ibid.), la práctica en comunidad de investigación (Daniel, Lafortune, Pallascio y Schleifer, 2000, en Ibid.), o la validez de los métodos de evaluación de estudiantes empleados en clase (Sanz de Acedo e Iriarte, 2001, en Ibid.). ${ }^{4}$

Entre los pocos estudios realizados en el campo de la educación moral según la propuesta de FpN, está el de Casado y Sánchez-Gey (2000, en Romero, 2004), realizado sobre dos muestras de estudiantes universitarios en Formación del profesorado, y la investigación de Daniel y Schleifer (2008), sobre las relaciones entre el diálogo en comunidad de investigación y la prevención de la violencia, así como el trabajo de Gagnon, Yergeau y Couture (2013) sobre el aprendizaje de la convivencia (vivre ensemble en el original en francés) a través de la práctica del diálogo filosófico en el aula. En cualquier caso, si bien hay algunos estudios realizados, estamos lejos de poder apoyar con la investigación científica la validez del programa de FpN como programa de educación moral ${ }^{5}$.

El segundo foco de críticas que añadimos a los cuatro señalados por Daniel (Ibid.) se refiere a los materiales de $\mathrm{FpN}$ específicamente pensados para la educación moral. Si bien, de las ocho novelas que componen el currículum básico de FpN, dos de ellas, Lisa (Lipman, 1993/2011) y Nous (Lipman, 1997/2004), así como las guías de uso que las acompañan, promueven directamente la investigación ética, es deseable contar en $\mathrm{FpN}$ con otros materiales que aborden la vertiente ética de cuestiones socialmente vivas en la actualidad. En este sentido, trabajos como los realizados sobre educación cosmopolita y FpN en el marco del programa PEACE (Agundez-Rodriguez, 2017a), o nuestro trabajo de investigación y elaboración de materiales de $\mathrm{FpN}$ para la educación ecosocial y del consumo (Agundez-Rodriguez, 2017b y 2018) pueden servir de ejemplos de la progresión que debe seguir la elaboración de materiales de $\mathrm{FpN}$ para la práctica de la educación moral hoy en día.

\section{conclusión}

Tomada en su apuesta de educación moral, la $\mathrm{FpN}$ es una propuesta integradora y original. Integradora, en el sentido de que es una propuesta que reúne los elementos esenciales presentes en los principales enfoques contemporáneos de la educación. Original, por promover el desarrollo de un pensamiento de orden superior, crítico, creativo y cuidante, como necesario a la formación permanente del sujeto moral. Además, esta consonancia con dichos

\footnotetext{
${ }^{4}$ Los estudios mencionados por Mortier (2008) deben ser tomados como ejemplos de evaluación del programa de $\mathrm{FpN}$. En ningún caso se trata de una enumeración exhaustiva de todos los estudios realizados. Además, desde 2008 otros estudios han sido realizados en este sentido. Sin embargo, consideramos que no es pertinente presentarlos aquí de manera exhaustiva al no tratarse de un texto centrado en la evaluación del programa de FpN.

5 Tampoco al enumerar estos trabajos sobre el programa de FpN como programa de educación moral pretendemos ser exhaustivos en nuestra exposición.
} 
programa de filosofía para niños como propuesta de educación moral: análisis comparado con otros enfoques de la educación moral

enfoques es lo que confiere al programa de $\mathrm{FpN}$ su valía y su gran actualidad como propuesta de educación moral.

En un intento de resumir la aproximación lipmaniana a la educación moral, podemos decir, junto al enfoque de la Clarificación de valores, que es una propuesta holística que demanda la intervención de todas las capacidades del sujeto moral. Del mismo modo, descubrimos los siguientes aspectos en el corazón de la educación moral que Lipman propone: al lado del enfoque de la solicitud, la importancia del Otro en la construcción del yo moral; cerca del enfoque narrativo, la fuerza y necesidad de la narración vital del Otro en la elaboración de la historia personal; junto a la ética discursiva, la relevancia del diálogo y de la argumentación; y, por último, en concierto con las éticas cordial y de la reconstrucción, encontramos también en $\mathrm{FpN}$ el corazón (la cordialidad, la afectividad) en ese corazón de la educación moral.

Además, también en común con todos estos enfoques contemporáneos, en FpN se entiende la intersubjetividad como elemento clave de la educación moral. En la propuesta de Lipman, este hecho se actualiza de manera permanente en la comunidad de investigación ética, como escenario donde se concretiza el desarrollo del pensamiento de orden superior (crítico, creativo y cuidante), elemento de la propuesta lipmaniana (especialmente en su calidad de cuidante) que le confiere originalidad.

Esta gran integralidad y originalidad son las que dotan a la propuesta ética de FpN de una gran complejidad, que es a la vez su mayor fuerza y su máxima debilidad: mientras por un lado le confieren una enorme riqueza y una constatada validez, por otro lado dificultan su puesta en práctica. Sólo una sólida formación del profesorado encargado de practicar $\mathrm{FpN}$ en su vertiente de educación moral, puede asegurar el éxito de su aplicación. Además, al hilo de nuestro estudio, sugerimos la ampliación de investigaciones científicas que vengan a reafirmar la validez de la propuesta lipmaniana para la educación moral, así como la creación de nuevos materiales con contenido ético, que faciliten la puesta en práctica del programa para tratar cuestiones socialmente vivas en la actualidad. Estos dos aspectos señalados abren dos vías interesantes de investigación futura en el campo de la educación moral asociada al programa de Matthew Lipman.

\section{referencias}

Agundez-Rodriguez, A. (2018). Éducation relative à la consommation au primaire selon l'approche de philosophie pour enfants. Revue Éducation relative à l'environnement, 13(2). Disponible en: http://journals.openedition.org/ere/865

Agundez-Rodriguez, A. (2017a). Éducation relative à la consommation : une dimension de l'écocitoyenneté. En Sauvé, L., Orellana, I. Bader, B. y Villemagne, C. (dir.), Vivre ensemble ici: repères contemporaines pour l'éducation, p. 198-216. Québec: PUQ.

Agundez-Rodriguez, A. (2017b). Filosofía para niños y educación cosmopolita: resultados de una investigación europea. Pensar Juntos, 1, p. 55-67. 
Apel, K-O. y Dussel, E. (2005). Ética del discurso; ética de la liberación. Madrid: Trotta.

Bauman, Z. (2006). Ética posmoderna. (2a ed.). Mexico: s. xxi. (1 ${ }^{\mathrm{a}}$ ed. 2005).

Bouchard, N. (2000). L'éducation morale à l'école. Une approche par le jeu dramatique et l'écriture. Québec: Presses de l'Université de Québec.

Bouchard, N. (2002). L'approche reconstructive. En Bouchard, N., Pour un renouvellement des pratiques d'éducation morale (p. 171-196). Québec: Presses de l'Université de Québec.

Caron, A. (2002). Préface. En Bouchard, N., Pour un renouvellement des pratiques d'éducation morale (p. IX-XI). Québec: Presses de l'Université de Québec.

Cortina, A. (1986). Ética mínima: introducción a la filosofía práctica. Madrid: Tecnos.

A. (1990). Ética sin moral. Madrid: Tecnos.

A. (1994). La ética de la sociedad civil. Madrid: Anaya.

A. (1995). Ética civil y religión. Madrid: PPC.

A. (1997). Ciudadanos del mundo: hacia una teoría de la ciudadanía. Madrid : Alianza.

A. (2007). Ética de la razón cordial. Oviedo: Ediciones Nobel.

Daniel, M.F. (1992). La philosophie et les enfants. Montréal : Logiques.

. M.F. y Schleifer (2008). L'apprentissage du dialoguer et la prévention primaire de la violence. En La philosophie pour enfants (p. 231-246). Bruselas: De Boeck.

Dewey, J. (1964). Naturaleza humana y conducta. México: Fondo de cultura económica. (Obra original publicada en 1922).

. J. (1969). Moral principles in education. New York: Greenwood Press. (Obra original publicada en 1909).

1916).

J. (2004a). Democracia y educación. Madrid: Morata. (Obra original publicada en

J. (2004b). Experiencia y educación. Madrid: Biblioteca Nueva. (Obra original publicada en 1938).

Escámez, J. (1998). La educación moral. En Gil Colomer (coord.), Filosofía de la educación, hoy (p. 303-320). Madrid: Dykinson.

Fullat, O. (2004). Axiología educativa para Occidente. En Torres, M. y Gutiérrez, A.E. (coord.), Tradición y valores en la posmodernidad (p. 19-68). México: Instituto tecnológico y de estudios superiores de la Universidad de Monterrey.

Gagnon, M., Yergeau, S. y Couture, E. (2013). L'apprentissage du vivre ensemble par la pratique du dialogue philosophique en classe : propos d'adolescents. Revue des sciences de l'éducation de McGill/McGill Journal of education, 41(1), p. 57-78.

García Moriyón, F. (1998). La escuela como ámbito de la educación moral. En VVAA, La formación moral de la juventud (p. 41-67). Madrid: Bruño.

. F. (2002). La estimulación de la inteligencia. Madrid: Ediciones de la Torre.

Gauchotte, P. (1992). Le pragmatisme. Paris: Presses universitaires de France.

Gendron, C. (2002). La sollicitude. En Bouchard, N., Pour un renouvellement des pratiques d'éducation morale (p. 47-84). Québec: Presses de l'Université de Québec.

Gervilla, E. (1998). Educación y valores. En Gil Colomer (coord.), Filosofía de la educación, hoy (p. 397-426). Madrid: Dykinson.

Gilligan, C. (1986). Une si grande différence. Paris : Flammarion.

Habermas, J. (2000). Aclaraciones a la ética del discurso. Madrid: Trotta.

. J. (2008). Conciencia moral y acción comunicativa. Madrid: Trotta.

Hegel, G.W.F. (2006). Fenomenología del espíritu. Valencia: Pre-textos. (Obra original publicada en 1807).

Hersh, R., Reimer, J. y Paolitto, D. (2002). El crecimiento moral. De Piaget a Kohlberg. Madrid:

Narcea.

Horkheimer, M. (2000). Anhelo de justicia. Madrid: Trotta.

childhood \& philosophy, rio de janeiro, v. 14, n. 31, set.-dez. 2018, pp. 671-695 issn 1984-5987 
programa de filosofía para niños como propuesta de educación moral: análisis comparado con otros enfoques de la educación moral

Kant, E. (2005). Crítica de la razón pura. México: Porrúa. (Obra original publicada en 1781). Kohlberg, L. (1984). The psychology of moral development: the nature and validity of moral stages. San Francisco: Harper \& Row.

Lago, J-C. (2006). Redescribiendo la comunidad de investigación. Pensamiento complejo y exclusión. Madrid : Ediciones de la Torre.

Laprée, R. (2002). La Clarification des valeurs "nouvelle manière". En Bouchard, N., Pour un renouvellement des pratiques d'éducation morale (p. 1-46). Québec: Presses de l'Université de Québec.

Leleux, C. (2002). L'éthique de la discussion. En Bouchard, N., Pour un renouvellement des pratiques d'éducation morale (p. 105-134). Québec: Presses de l'Université de Québec.

Levinas, E. (2007). Humanismo del otro hombre. Madrid: s. xxi.

Lipman, M., Sharp, A.M. y Oscanyan, F-S. (1980). Philosophy in the Classroom. Philadelphia: Temple University Press.

M. y Sharp, A.M. (1988). Investigación ética. Manual del profesor para acompañar a Lisa. Madrid: Ediciones de la Torre.

. M. (1988). Philosophy goes to School. Philadelphia: Temple University Press.

. M. (1993). Lisa. Québec: Association québécoise de Philosophie pour enfants.

M. (1995). Caring as Thinking, Inquiry 15(1), 1-13.

M. (1997/2010). Nous. Madrid: Ediciones de la Torre.

de la Torre.

M., Sharp, A.M. y Oscanyan, F-S. (1998). La filosofía en el aula. Madrid: Ediciones

. M. (1998). Pensamiento complejo y educación. Madrid: Ediciones de la Torre.

M. (2000). Algunos supuestos filosóficos de filosofía para niños. En Kohan, W.O.

(comp.), Filosofía para niños (p. 61-64). Brasil: Novedades educativas.

. M. (2003). Thinking in education. ( $2^{\mathrm{a}}$ ed.). Cambridge : Cambridge University Press.

(1 $1^{\mathrm{a}}$ ed. 1991).

Miranda, T. (1991). J. Dewey: naturaleza humana y moral. Aprender a pensar 3(1), 7-15.

Morris, R. y Caron, A. (2002). L'approche narrative. En Bouchard, N., Pour un renouvellement des pratiques d'éducation morale (p. 135-170). Québec: Presses de l'Université de Québec.

Mortier, F. (2008). Études d'évaluation: la methode de Matthew Lipman comme moyen de développement. En La philosophie pour enfants (p. 47-70). Bruselas: De Boeck.

Nussbaum, M. (1990). Love's Knowledge: essays on Philosophy and Literature. New York: Oxford University Press.

Nussbaum, M. (1995). Poetic justice: the Literary Imagination and Public Life. Boston: Beacon Press.

Pérez de Tudela, J. (1988). El pragmatismo americano. Madrid: Cincel.

Puig Rovira, J.M. (2003). Prácticas morales: una aproximación a la educación moral. Barcelona: Paidós Ibérica.

Putnam, H. (1999). El pragmatismo: un debate abierto. Barcelona: Gedisa, 1999.

Raths, L.E., Harmin, M., Simon, S.B. (1978). Values and teaching. Working with Values in the Classroom. (2 ${ }^{\mathrm{a}}$ ed.). Columbus: Charles E. Merrill. (1 $1^{\mathrm{a}}$ ed., 1966).

Rawls, J. (2002). Justicia como equidad. Madrid: Tecnos.

Reboul, O. (1992). Les valeurs de l'éducation. Coll : Premier cycle. Paris : PUF.

Ricoeur, P. (2005). Caminos del reconocimiento. Madrid: Trotta.

694 childhood \& philosophy, rio de janeiro, v. 14, n. 31, set.-dez. 2018, pp. 671-695 issn 1984-5987 
Romero (2004). Efectos del programa Filosofía para niños y niñas en el clima social del aula. Alcalá de Henares: Universidad de Alcalá de Henares, Departamento de Educación. (Tesis doctoral).

Rorty, R. (1996). Consecuencias del pragmatismo. Madrid: Tecnos.

Roy, L. (2002). L'éducation du caractère. En Bouchard, N., Pour un renouvellement des pratiques d'éducation morale (p. 85-104). Québec: Presses de l'Université de Québec.

Rubio, J. (2000). Educación moral, postmodernidad y democracia. Madrid: Trotta.

Sasseville, M. (1994). Self-Esteem, Logical Skills and Philosophy for Children. Thinking 11(2), 30-37.

Savater, F. (1992). Ética para Amador. (13 a ed.). Barcelona: Ariel.

Sharp, A.M. (1990). ¿Qué es una comunidad de investigación filosófica? Aprender a pensar 2(2), 7-19.

Tozzi, M. (2008). Lipman, Lévine, Tozzi : différences et complémentarités. En La philosophie pour enfants (p. 96-116). Bruselas: De Boeck.

recibido en: 23.05 .2018

aprobado en: 20.08 .2018 